\title{
In vitro probiotic characteristics of Lactobacillus plantarum ZDY 2013 and its modulatory effect on gut microbiota of mice
}

\author{
Renhui Huang, ${ }^{*}$ Xueying Tao, ${ }^{*}$ Cuixiang Wan, ${ }^{*}$ Shengjie Li, ${ }^{*}$ Hengyi $X u,{ }^{*}$ Feng Xu, ${ }^{*}$ Nagendra P. Shah, $\dagger$ \\ and Hua Wei*1 \\ *State Key Laboratory of Food Science and Technology, Nanchang University, Nanchang 330047, P. R. China \\ †Food and Nutritional Science, School of Biological Science, The University of Hong Kong, Pokfulam Road, Hong Kong, China
}

\begin{abstract}
Lactobacillus plantarum ZDY 2013, a novel strain isolated from Chinese traditional fermented acid beans, was systematically evaluated for its survival capacity under stress conditions ( $\mathrm{pH}$, bile salt, simulated gastrointestinal tract, and antibiotics), production of exopolysaccharide and antagonism against 8 pathogens. Its effect on mice gut microbiota was also investigated by quantitative PCR and PCR-denaturing gradient gel electrophoresis. The results showed that ZDY 2013 can grow at $\mathrm{pH} 3.5$ and survive at $\mathrm{pH} 2.0$ for $6 \mathrm{~h}$ and at $0.45 \%$ bile salt for $3 \mathrm{~h}$. The exopolysaccharide yield was up to $204 \pm 7.68 \mathrm{mg} / \mathrm{L}$. The survival rate of ZDY 2013 in a simulated gastrointestinal tract was as high as $65.84 \%$. Antagonism test with a supernatant of ZDY 2013 showed maximum halo of $28 \mathrm{~mm}$ against Listeria monocytogenes. The inhibition order was as follows: Listeria monocytogenes, Salmonella typhimurium, Escherichia coli, Pseudomonas aeruginosa, Shigella sonnei, Enterobacter sakazakii, and Staphylococcus aureus. Lactobacillus plantarum ZDY 2013 was sensitive to some antibiotics (e.g., macrolide, sulfonamides, aminoglycoside, tetracyclines and $\beta$-lactams), whereas it was resistant to glycopeptides, quinolones, and cephalosporins antibiotics. Denaturing gradient gel electrophoresis profile demonstrated that ZDY 2013 administration altered the composition of the microbiota at various intestinal loci of the mice. Moreover, the quantitative PCR test showed that the administration of ZDY 2013 enhanced the populations of Bifidobacterium and Lactobacillus in either the colon or cecum, and reduced the potential enteropathogenic bacteria (e.g., Enterococcus, Enterobacterium, and Clostridium perfringens). Lactobacillus plantarum ZDY 2013 exhibited high resistance against low $\mathrm{pH}$, bile salt, and gastrointestinal fluid, and possessed antibacterial and gut microbiota modulation
\end{abstract}

Received November 25, 2014.

Accepted May 5, 2015.

${ }^{1}$ Corresponding authors: weihua114@live.cn and npshah@hku.hk properties with a potential application in the development of dairy food and nutraceuticals.

Key words: Lactobacillus plantarum ZDY 2013, stress resistance, quantitative PCR, antagonistics

\section{INTRODUCTION}

The beneficial effects of probiotics (e.g., Lactobacillus and Bifidobacterium) have been documented by numerous investigations over many years, and mainly included improvement in the health of intestinal tract, enhancement of immune system, reduction of intestinal disorders, and risk of certain cancers (Salminen et al., 1998; Parvez et al., 2006; Jankovic et al., 2010). Generally, it has been assumed that probiotics, being of intestinal origin, can be used for human or animal consumption for exerting beneficial effects on host health through the interaction with host and for providing positive influence in the alleviation or prevention of diverse intestinal disorders (Borriello et al., 2003). For example, Lactobacillus rhamnosus GG, one of the most successful commercial Lactobacillus strains isolated from human gastrointestinal tract, has been used for nutraceutical supplement in numerous microecological studies (García-Ródenas et al., 2006).

In addition to isolating probiotics organisms from human feces, traditional fermented foods have been another important source for isolating probiotic organisms in many countries (ben Omar and Ampe, 2000; Tamime, 2002; Cheng et al., 2014). Isolation of probiotic organisms and evaluation of their properties has attracted a lot of attention in recent decades. In this context, isolation of Lactobacillus plantarum is important, as it is found in traditional fermented food, especially in fermented foods from Korea, China, and India (Salminen et al., 1998; Jankovic et al., 2010). Kleerebezem et al. (2003) carried out the complete genome sequence $L$. plantarum WCFS1, which was isolated from kimchi, and annotated the interaction of this organism with its environment at molecular level. However, most of the work on L. plantarum focused on its antioxidant, antimutagen, modulation of immune system, lowering 
cholesterol level, and reducing lactose-intolerance capabilities (Naidu et al., 1999; Fooks and Gibson, 2002; Gill, 2004; Parracho et al., 2007). The behavior of $L$. plantarum is diverse depending on different sources of isolation. Some isolates developed resistance to extreme acidic conditions, whereas others can survive in high concentration of bile salt (Liong and Shah, 2005; Guidone et al., 2014). Kaushik et al. (2009) reported that L. plantarum LP9 survived at $\mathrm{pH} 1.5$ to 2.0 or in 1.5 to $2.0 \%$ oxgall bile for $2 \mathrm{~h}$. Similarly, Lee et al. (2011) reported that L. plantarum CJLP133, isolated from kimchi, could survive at $\mathrm{pH} 2.5$ for $3 \mathrm{~h}$ and in $0.3 \%$ oxgall for $24 \mathrm{~h}$. Therefore, characterization of isolates of $L$. plantarum from traditional fermented food is important to ascertain the mechanism of probiotic action for promoting human health. Very limited information exists on in vivo interaction of $L$. plantarum with intestinal microbiota. In our study, a novel strain, L. plantarum ZDY 2013, was isolated from Chinese home-made acid beans and was systematically assessed for its survival in stress conditions $(\mathrm{pH}$, bile salt, mimic gastrointestinal tract, and antibiotics) and in vivo interaction with indigenous microbial biota using quantitative real-time PCR (qPCR) combined with denaturing gradient gel electrophoresis (DGGE).

\section{MATERIALS AND METHODS}

\section{Bacterial Strains Isolation and Identification}

Traditional acid beans were collected from a village in Fujian province, China, and fermented under natural conditions without any additional bacteria. The bacterial strains were isolated from acid beans as described herein. Acid beans were collected from local village in Fujian province, China. Bacteria were isolated from acid beans according to a modified method described before (Wei et al., 2006; Wu et al., 2009). Briefly, $10 \mathrm{~g}$ of fermentation broth and beans were added to $90 \mathrm{~mL}$ of sterile PBS buffer, followed by homogenization. The suspension was serially diluted $\left(10^{-1}, 10^{-2}, 10^{-3}\right)$ and $0.1 \mathrm{~mL}$ of each dilution was surface plated in duplicates on de Man, Rogosa, Sharpe (MRS) agar. The plates were incubated under anaerobic conditions at $37^{\circ} \mathrm{C}$ for $48 \mathrm{~h}$. Colonies with distinct morphological differences, such as color, shape, and size, were selected and purified by streaking 3 times on MRS agar and were kept in MRS-agar stabs at $4^{\circ} \mathrm{C}$, and frozen in $30 \%$ glycerol solution and stored at $-80^{\circ} \mathrm{C}$. A representative isolate was amplified by PCR method using the $16 \mathrm{~S}$ rRNA universal primer. The amplicons were sequenced by Sangon Biotech, Ltd. (Shanghai, China) and the sequences were searched against NCBI database (http:// www.ncbi.nlm.nih.gov/index.html) using the BLASTN algorithm. Based on these, the organism was classified as L. plantarum and was given the strain no. ZDY 2013.

\section{Acid and Bile Salt Tolerance Properties}

The isolate L. plantarum ZDY 2013 was grown in sterile MRS broth at $37^{\circ} \mathrm{C}$ for $24 \mathrm{~h}$, and centrifuged at $7,500 \times g$ for $10 \mathrm{~min}$ at $4^{\circ} \mathrm{C}$. The cell pellet was diluted $100 \times$ in sterile MRS broth and the $\mathrm{pH}$ was adjusted to $6.5,5.0,4.0$, and 3.5 for acid adaptation; another set of media was adjusted to 3.0 and 2.0 for acid challenge studies. Bile salt tolerance was performed in the medium supplemented with $0,0.15,0.30$, and $0.45 \%$ (wt/ vol) bile salts (Yuanye Bio-Technology Ltd., Shanghai, China). Freshly prepared cultures were incubated at $37^{\circ} \mathrm{C}$. The absorbance at $600 \mathrm{~nm}$ and $\mathrm{pH}$ values of fermentation cultures were measured immediately and at every $2 \mathrm{~h}$. Viable counts were enumerated after acid challenge and bile salt tolerance test at $0,3,6$, and 12 h.

\section{Exopolysaccharide Yield}

The MRS broth $(100 \mathrm{~mL})$ was inoculated with $1 \%$ ZDY 2013 and cultured at $37^{\circ} \mathrm{C}$ in $250-\mathrm{mL}$ Erlenmeyer flasks. Aliquots $(5 \mathrm{~mL})$ were withdrawn at various time points to determine the viable cell counts and exopolysaccharide (EPS) yield. The EPS from cell-free culture supernatant was extracted and detected by the methods as described by Li et al. (2014a). Briefly, bacterial cells were removed by centrifugation at $4,000 \times g$ for 10 min at $4^{\circ} \mathrm{C}$, and the cell-free culture supernatant was treated with ethanol overnight at $4^{\circ} \mathrm{C}$ followed by centrifugation $\left(8,000 \times g, 20 \mathrm{~min}, 4^{\circ} \mathrm{C}\right)$. The precipitate was dissolved in $5 \mathrm{~mL}$ of distilled water. Proteins were removed using Sevage agent (chloroform: n-butanol; $4: 1)$ followed by centrifugation $\left(8,000 \times g, 10 \mathrm{~min}, 4^{\circ} \mathrm{C}\right)$. The supernatant was removed to another receptacle and precipitated with ethanol again. The precipitated EPS was dissolved with distilled water; EPS concentration was determined by the phenol-sulfuric acid assay method (Li et al., 2014a).

\section{Antimicrobial Activities of ZDY 2013}

Enterobacter sakazakii ATCC 29544, Escherichia coli O 157: H 7, Salmonella typhimurium ATCC 13311, Shigella sonnei ATCC 25931, Bacillus cereus ATCC 14579, Pseudomonas aeruginosa MCC 10104, Listeria monocytogenes CMCC 54007, and Staphylococcus aureus CMCC 26003 were selected to test the antibacterial activities of $L$. plantarum ZDY 2013. The antibacterial activity was investigated by agar diffusion assay as described previously by Chen et al. (2014b) with 
a slight modification. Overnight-incubated culture of the indicator microorganisms was adjusted to $10^{6}$ to $10^{7} \mathrm{cfu} / \mathrm{mL}$ and $100-\mu \mathrm{L}$ aliquots of the organisms were spread on Luria-Bertani agar; then $200 \mu \mathrm{L}$ of cell-free supernatant of ZYD 2013 was added into the oxford cup (a stainless steel cylinder of outer diameter 7.8 $\pm 0.1 \mathrm{~mm}$, inner diameter $6.0 \pm 0.1 \mathrm{~mm}$, and height $10.0 \pm 0.1 \mathrm{~mm}$ ) which was placed on the surface of the agar. The diameter of the inhibition zone around the cup (including that of the Oxford cup, $7.8 \mathrm{~mm}$ ) was measured after $12 \mathrm{~h}$ of incubation. The experiment was performed in triplicate.

\section{Antibiotic Sensitivity Test}

The disk-diffusion method was used to determine the antibiotic susceptibility of ZDY 2013 according to Tan et al. (2013). Briefly, the organism was inoculated into MRS broth and incubated at $37^{\circ} \mathrm{C}$ overnight, $100 \mu \mathrm{L}$ of diluted culture $\left(10^{7} \mathrm{cfu} / \mathrm{mL}\right)$ was spread onto MRS agar, and the antibiotic discs were applied onto the surface. The concentrations of indicator antibiotics are shown in Table 1. Plates were incubated at $37^{\circ} \mathrm{C}$ and evaluated after $24 \mathrm{~h}$. Inhibition zones around the discs were measured using a digital caliper.

\section{Simulated Gastrointestinal Transit}

The survival of ZDY 2013 in the simulated gastrointestinal tract (GIT) was studied in a simulation model with some modifications (Salazar et al., 2011, Tan et al., 2013). To simulate the GIT transit, the bacterial suspension was submitted to the gastric juice (GJ ; con- taining $3 \mathrm{~g} / \mathrm{L}$ of pepsin dissolved with PBS, pH 3.0), duodenum juice [DJ; containing 1\% (wt/vol) bile salt, $\mathrm{pH}$ 8.0], and intestinal juice [IJ ; containing $0.3 \%$ (wt/ vol) bile salt, $0.1 \%$ (wt/vol) pancreatin], sequentially. The bacterial cells were harvested by centrifugation $\left(7,500 \times g, 5 \mathrm{~min}, 4^{\circ} \mathrm{C}\right)$ after $24 \mathrm{~h}$ of incubation and washed twice with PBS, and then a 1.0-mL suspension was centrifuged $\left(7,500 \times g, 5 \mathrm{~min}, 4^{\circ} \mathrm{C}\right)$ and resuspended in $10 \mathrm{~mL}$ of GJ. Finally, bacterial suspension was incubated for $90 \mathrm{~min}$ at $37^{\circ} \mathrm{C}$ with shaking (100 rpm). Afterward, cells were harvested, resuspended in DJ, and incubated under static conditions for $10 \mathrm{~min}$ at $37^{\circ} \mathrm{C}$ in anaerobic condition. Finally, cells were harvested again, resuspended in IJ, and incubated for $120 \mathrm{~min}$ at $37^{\circ} \mathrm{C}$ in anaerobic condition. Viable counts were enumerated before each step and at the end of GIT transit.

\section{Animal Model and Experiment Design}

The protocol for carrying out animal experiments was approved by Nanchang University Animal Ethics Committee and all the ethical requirements to conduct the experiment were met. All procedures were conducted in compliance with protocols provided and approved by the Animal Care Review Committee (approval number 0064257) of Nanchang University (Jiangxi, China). Twenty 6- to 8-wk-old BALB/c mice obtained from Nanchang University Animal Science Department were used in the modulation of intestinal microbiota study after oral administration of ZDY 2013. The mice were acclimatized for $1 \mathrm{wk}$ and assigned into their respective groups randomly (10 mice each group) based on baseline BW. Mice were gavaged twice a day (with an

Table 1. Antibiotic susceptibility of Lactobacillus plantarum ZDY 2013

\begin{tabular}{|c|c|c|c|c|}
\hline Class & Antibiotic & $\begin{array}{l}\text { Content } \\
(\mu \mathrm{g})\end{array}$ & $\begin{array}{c}\text { Diameter of } \\
\text { inhibition zone } \\
(\mathrm{mm})\end{array}$ & Sensitivity $^{1}$ \\
\hline Macrolide & Erythromycin & 15 & $33.13 \pm 0.85$ & $\mathrm{~S}$ \\
\hline \multirow{2}{*}{ Sulfonamides } & Rifampicin & 5 & $21.75 \pm 0.29$ & $\mathrm{~S}$ \\
\hline & Sulphamethoxazole & 25 & $37.25 \pm 0.96$ & $\mathrm{~S}$ \\
\hline \multirow{3}{*}{ Aminoglycoside } & Streptomycin & 10 & $16.5 \pm 0.58$ & $\mathrm{~S}$ \\
\hline & Gentamicin & 10 & $21.50 \pm 0.58$ & $\mathrm{~S}$ \\
\hline & Kanamycin & 30 & $17.00 \pm 0.82$ & I \\
\hline Nitrofurans & Nitrofurantoin & 300 & $32.63 \pm 1.11$ & $\mathrm{~S}$ \\
\hline Tetracyclines & Tetracycline & 30 & $25.88 \pm 1.03$ & $\mathrm{~S}$ \\
\hline Amphenicols & Chloramphenicol & 30 & $28.75 \pm 0.96$ & $\mathrm{~S}$ \\
\hline \multirow[t]{2}{*}{ Beta-lactams } & Amoxicillin & 10 & $28.25 \pm 0.87$ & $\mathrm{~S}$ \\
\hline & Penicillin & $10 \mathrm{U}$ & $28.88 \pm 0.25$ & I \\
\hline \multirow[t]{2}{*}{ Glycopeptide } & Bacitracin & 0.04 & $6.17 \pm 0.29$ & $\mathrm{R}$ \\
\hline & Polymyxin B & 30 & $7.25 \pm 0.29$ & $\mathrm{R}$ \\
\hline \multirow[t]{2}{*}{ Quinolones } & Ciprofloxacin & 5 & $10.75 \pm 0.96$ & $\mathrm{R}$ \\
\hline & Nalidixic acid & 30 & $6.63 \pm 0.63$ & $\mathrm{R}$ \\
\hline Cephalosporins & Cefoxitin & 30 & $14.00 \pm 0.82$ & $\mathrm{R}$ \\
\hline
\end{tabular}

${ }^{1} \mathrm{~S}=$ susceptible; $\mathrm{I}=$ intermediate $; \mathrm{R}=$ resistance. 
interval of $8 \mathrm{~h}$ ) for $2 \mathrm{wk}$. Each mouse in the treatment group received $200 \mu \mathrm{L}$ of sterile PBS containing $10^{7}$ to $10^{8} \mathrm{cfu} / \mathrm{mL}$ of ZYD 2013 cells, whereas mice in the control group received an equal volume of sterile PBS. Activity, behavior, fur health, and live weight were observed twice daily during the experimental period. Twenty-four hours after the final administration, mice were euthanized by $\mathrm{CO}_{2}$ inhalation followed by cervical dislocation. Blood samples were collected for chemical analysis, as discussed below. The kidneys, spleen, and thymus were weighed and their weights relative to the final BW (organ index) were calculated. Fecal pellets, cecum contents, and colon were also collected and stored at $-20^{\circ} \mathrm{C}$ until analyzed.

\section{Health Markers of Mice}

Blood samples (approximately $0.8 \mathrm{~mL}$ ) were taken from orbital sinus by quickly removing the eyeball the control and L. plantarum ZDY 2013 treatment groups (6 mice per group) after euthanizing them. Ten important hepatic markers were then measured, including alanine aminotransferase, aspartate aminotransferase, total bilirubin, direct bilirubin, total protein, albumin, globulin, ratio of albumin to globulin, gamma glutamyl transaminase, alkaline phosphatase, creatinine, BUN, and urea. These various serum biochemical markers were indicators of liver and kidney functions. Blood samples were centrifuged $\left(4,000 \mathrm{rpm}, 10 \mathrm{~min}, 4^{\circ} \mathrm{C}\right)$ to obtain at least $0.25 \mathrm{~mL}$ of blood plasma for analysis of serum biochemistry using biochemical analyzer at the First Affiliated Hospital of Nanchang University, Nanchang, China (Yang et al., 2015).

\section{qPCR Assays}

The genomic DNA of intestinal contents and fecal samples were extracted using the bead-beating method (Li et al., 2014a). The qPCR amplification was performed using an ABI $7900 \mathrm{HT}$ fast real-time PCR system (Applied Biosystems, Carlsbad, CA) and the reaction of $\mathrm{qPCR}$ assays contained $10 \mu \mathrm{L}$ of SYBR Primer EX TaqII (Takara, Dalian, China), 0.4 $\mu \mathrm{L}$ of ROX Reference Dye (50×; Takara), $1.0 \mu \mathrm{L}$ of DNA template, and $0.8 \mu \mathrm{L}$ of each of the primers (the final concentration was $0.4 \mu M$ ) with $7 \mu \mathrm{L}$ of milli-Q $\mathrm{H}_{2} \mathrm{O}$ (Millipore, Billerica, MA). The amplification was programed to start at $95^{\circ} \mathrm{C}$ for $30 \mathrm{~s}$, followed by 40 cycles (degeneration at $95^{\circ} \mathrm{C}$ for $5 \mathrm{~s}$, annealing at $60^{\circ} \mathrm{C}$ for $30 \mathrm{~s}$; Wang et al., 2014). The relative population (fold change) of the target bacterial was analyzed using the $2^{-\Delta \Delta \mathrm{Ct}}$ method based on qPCR data according to Feng et al. (2010). Different groups and species of bacteria targeted by primers are listed in Table 2 .

\section{PCR-DGGE Analysis}

The V3 region of the $16 \mathrm{~S}$ rRNA gene was amplified using primers338 (F) with GC clamp and $519(\mathrm{R})$ followed by DGGE analysis, as described previously (Chen et al., 2014a; Li et al., 2014a). Briefly, the reaction mixture contained $25 \mu \mathrm{L}$ of $2 \times$ Taq MasterMix (CWBIO, Beijing, China), $0.1 \mu M$ of each primer, 1.0 $\mu \mathrm{L}$ of DNA template, and milli-Q $\mathrm{H}_{2} \mathrm{O}$ was added to obtain a final volume $50 \mu \mathrm{L}$. The PCR reaction began with 1 cycle of $5 \mathrm{~min}$ at $94^{\circ} \mathrm{C}$, followed by 30 cycles of $30 \mathrm{~s}$ at $94^{\circ} \mathrm{C}, 30 \mathrm{~s}$ at $56^{\circ} \mathrm{C}$, and $45 \mathrm{~s}$ at $72^{\circ} \mathrm{C}$, and a

Table 2. Primers (forward, F, and reverse R) used in this study ${ }^{1}$

\begin{tabular}{lll}
\hline Target organism & Oligonucleotide sequence $\left(5^{\prime}-3^{\prime}\right)$ & Reference \\
\hline V3 region & F: TACGGGAGGCAGCAG with GC clamp & Li et al., 2014a \\
& R: ATTACCGCGGCTGCTGG & \\
Total bacterial & F: ACTCCTACGGGAGGCAGCAG & Bartosch et al., 2004 and Li et al., 2014a \\
Bifidobacterium spp. & R: ATTACCGCGGCTGCTGG & Rinttilä et al., 2004 \\
Enterococcus spp. & R: TCGCGTCCGGTGTGAAAG & \\
& F: CCCTTATTGTTAGTTGCCATCAT & Rinttilä et al., 2004 \\
Fusobacterium spp. & R: ACTCGTTGTACTTCCCATTGT & Rinttilä et al., 2004 \\
Enterobacteriaceae & F: CCCTTCAGTGCCGCAGT & \\
& R: GTCGCAGGATGTCAAGAC & Bartosch et al., 2004 \\
Lactobacillus spp. & F: CATTGACGTTCCCGCAGAAGAAG & Rinttilä et al., 2004 \\
Bacteroides spp. & R: CTCTACGAGACTCAAGCTTGC & Rinttilä et al., 2004 \\
Clostridium perfringens & R: AGCAGTAGGGATGGAG & Wise and Siragusa, 2005 \\
& F: GGTGTCGGCTTAAGTGCCAT & \\
\hline
\end{tabular}

${ }^{1}$ GC clamp $=$ TACGGGAGGCAGCAG. 
final extension at $72^{\circ} \mathrm{C}$ for $10 \mathrm{~min}$. After amplification, the amplicons were separated by DGGE using the Dcode System (Bio-Rad, Hercules, CA; Chen et al., 2013) which was performed on $8 \%$ (wt/vol) polyacrylamide gels in running buffer containing $1 \times$ TAE [20 $\mathrm{mmol} / \mathrm{L}$ Tris, $10 \mathrm{mmol} / \mathrm{L}$ acetate, $0.5 \mathrm{mmol} / \mathrm{L}$ EDTA $(\mathrm{pH} 8.0)$ and a denaturing gradient of $35-65 \%$ urea and formamide (Sigma-Aldrich, St. Louis, MO), where $100 \%$ is defined as $7 \mathrm{~mol} / \mathrm{L}$ of urea and $40 \%$ ( $\mathrm{vol} / \mathrm{vol}$ ) formamide]. Electrophoresis was conducted under $80 \mathrm{~V}$ at $60^{\circ} \mathrm{C}$ for $16 \mathrm{~h}$. The DNA bands in gels were visualized by silver staining (Chen et al., 2012).

The DGGE patterns were subsequently normalized and analyzed using Bio-Rad Quantity One 4.4.0 software (Chen et al., 2011). The degree of similarity was described by a similarity coefficient. Digitalized DGGE patterns were analyzed by the principal component analysis (PCA) to change the microbiota into dots pattern. Richness indices were calculated according to equations previously described by Luo et al. (2004).

\section{Statistical Analysis}

Triplicate data were represented by mean \pm standard deviation. We used SPSS 13.0 software to perform statistical analyses (SPSS Inc., Chicago, IL) by means of independent one-way ANOVA tests. The multiple comparison of each test was assessed by least significant difference test $(P<0.05)$.

\section{RESULTS}

\section{Resistance to Acid and Bile Salts}

Several strains of Lactobacillus were verified by PCR and sequenced from collections of isolates from Chinese traditional acid beans. Moreover, the first run evaluation of survival rate under extreme conditions (e.g., low $\mathrm{pH}$, high concentration of bile salts) was performed and 1 strain with outstanding stress-resistant ability, $L$. plantarum ZDY 2013, was tested further for its sensitive to acid and bile salts. As shown in Figure 1, ZDY 2013 grew in a wide range of $\mathrm{pH}$, from 6.5 to 3.5 , although a minor difference in viability among groups was noted at $\mathrm{pH} 3,3.5$, and 4.0 (Figure 1A). To ascertain the survival of L. plantarum ZDY 2013 in extreme acidic conditions, a long-term resistant testing of viable cell counts (initial value of $2.24 \times 10^{7} \mathrm{cfu} / \mathrm{mL}$ ) in low $\mathrm{pH}$ was performed. As shown in Figure $1 \mathrm{~B}$, the bacterial count did not change significantly at $\mathrm{pH} 3.0$, or even after $6 \mathrm{~h}$ in $\mathrm{pH} 2.0$, but decreased significantly after 12 $\mathrm{h}$ in $\mathrm{pH}$ 2.0. To observe the effect of the initial $\mathrm{pH}$ of the original culture on L. plantarum ZDY 2013 fermentation, changes in $\mathrm{pH}$ values were tested for $36 \mathrm{~h}$. As shown in Figure 1C, the $\mathrm{pH}$ of the culture at $\mathrm{pH} 6.5$ and 5.0 decreased rapidly until $8 \mathrm{~h}$ and maintained this trend until $32 \mathrm{~h}$, and $\mathrm{pH} 4.0$ and 3.5 decreased slowly to a final $\mathrm{pH}$ of 3.2 ; however, only minor difference were noted among the various initial $\mathrm{pH}$ groups.

Considering common bile salt concentrations of human GIT range from 0.03 to $0.3 \%$, we used $0.15,0.30$, and $0.45 \%$ as target challenge concentrations. As shown in Figure 1D, the survival rate of cells of L. plantarum ZDY 2013 was nearly stable in all test groups at $3 \mathrm{~h}$; a reduction trend was observed in cell counts on increasing bile salt concentration. For example, the reduction in group of $0.45 \%$ was from $7.53 \times 10^{7}$ to $1.25 \times 10^{6}$ $\mathrm{cfu} / \mathrm{mL}$, whereas it was $1.78 \times 10^{7}$ and $5.01 \times 10^{6} \mathrm{cfu} /$ $\mathrm{mL}$, respectively, in 0.15 and $0.30 \%$ groups at $6 \mathrm{~h}$; the cell counts maintained a high level at $0.3 \%$ bile salt even after $12 \mathrm{~h}\left(1.01 \times 10^{6} \mathrm{cfu} / \mathrm{mL}\right)$.

\section{Growth and EPS Yield}

Among metabolic substances produced from L. plantarum ZDY 2013, it is likely that EPS may have contributed to the resistance against extreme acid environment. In our preliminary study, this Lactobacillus strain produced high amount of EPS among our collection of Lactobacillus strains (data not shown). As shown in Figure 2, the yield of EPS was positively related to the biomass of cells of L. plantarum ZDY 2013; the highest yield of EPS $(204 \pm 7.87 \mathrm{mg} / \mathrm{L})$ was achieved at 24 $\mathrm{h}$, indicating that EPS may have been a primary metabolite of L. plantarum ZDY 2013. Interestingly, the amount of EPS decreased during the stationary phase.

\section{Antagonistic Effect of Cell-Free Suspension of L. plantarum ZDY 2013 In Vitro}

Antagonistic effect against pathogens was the main probiotic trait for the evaluation of the potential probiotic organism. As shown in Figure 3, 8 different food-borne pathogens were significantly inhibited by the cell-free culture supernatant of $L$. plantarum ZYD 2013. Listeria monocytogenes CMCC 54007 was the most sensitive organism, with a halo as high as 28.33 $\pm 0.76 \mathrm{~mm}$, whereas $S$. aureus was the least sensitive, with a halo of $12.33 \pm 0.29 \mathrm{~mm}$.

\section{Antibiotic Sensitivity of L. plantarum ZDY 2013}

Considering potential application of Lactobacillus isolates together with a reasonable dose of antibiot- 
ics as combination therapies for intestinal disorder, antibiotic sensitivity of L. plantarum ZDY 2013 was evaluated. As shown in Table 2, susceptibility of $L$. plantarum ZDY 2013 varied among classes of antibiotics, it exhibited a steady resistance to glycopeptide (bacitracin, polymyxin B), quinolones (ciprofloxacin, nalidixic acid), and cephalosporins (cefoxitin), whereas ZDY 2013 was sensitive to macrolide (erythromycin), sulfonamides (rifampicin, sulphamethoxazole), aminoglycoside (streptomycin, gentamicin), tetracyclines, nitrofurans (nitrofurantoin), amphenicols (chloramphenicol), and $\beta$-lactams (e.g., amoxicillin), but showed an intermediate sensitivity to penicillin and kanamycin.

A

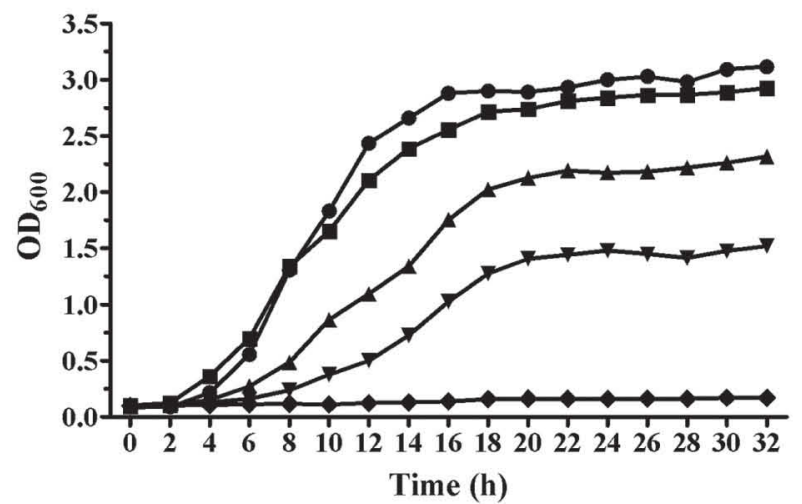

C

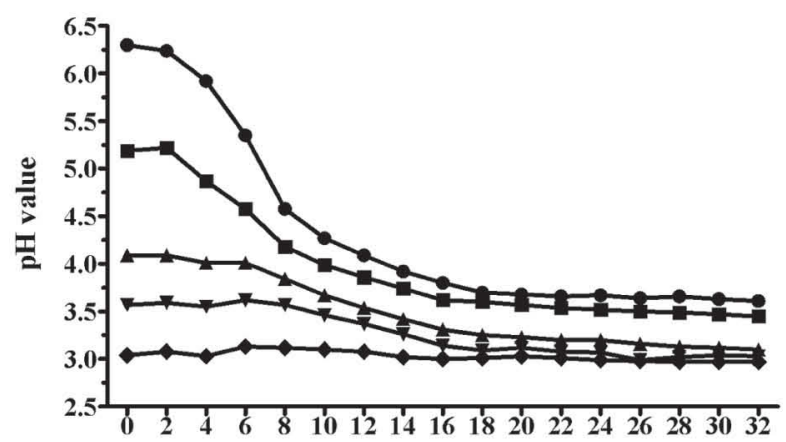

Time (h)

\section{Simulated Gastric-Duodenal-Intestinal Transit Testing}

Based on the results of extreme acid and high concentration bile salt resistance of L. plantarum ZDY 2013, we further constructed a simulated gastric-duodenalintestinal transit system, including mimic digestion enzyme (e.g., pepsin, pancreatin). As shown in Figure 4 , a gradual loss of cell counts was observed in the 3 phases of mimic digestion (i.e., GJ, DJ, and IJ). The final survival of cells achieved was as high as $65.84 \%$; the cell count was $6.31 \times 10^{7} \mathrm{cfu} / \mathrm{mL}$ after the complete gastric-duodenal-intestinal transit, with initial cell counts of $9.55 \times 10^{7} \mathrm{cfu} / \mathrm{mL}$ of L. plantarum ZDY 2013 .

\section{B}

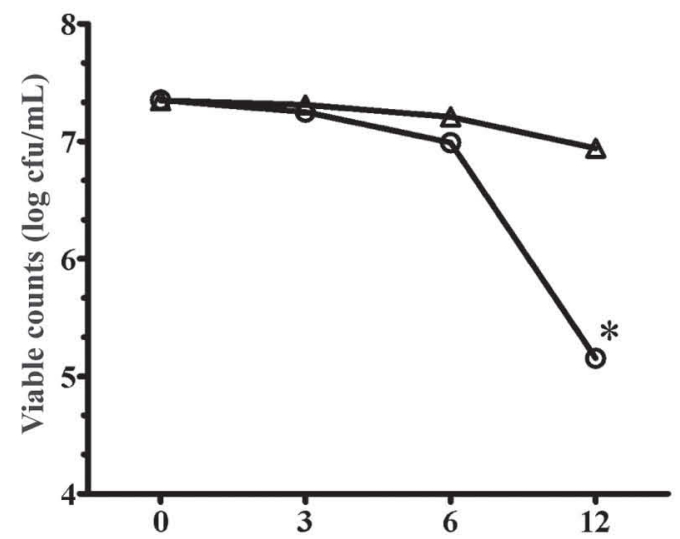

D

Time (h)

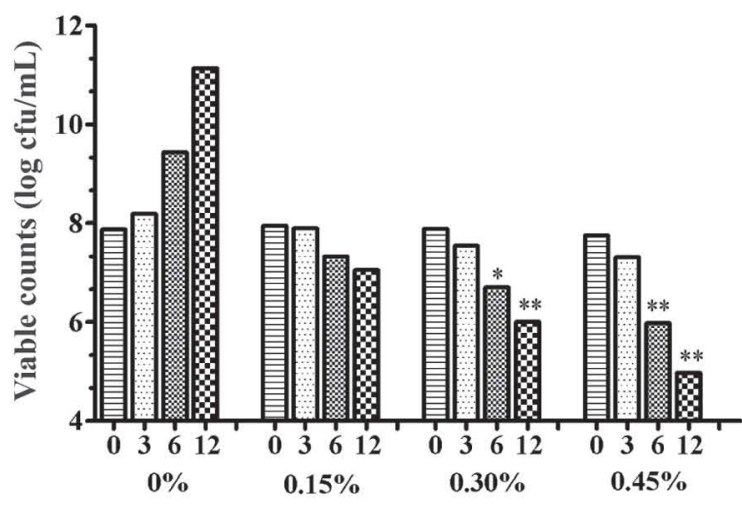

Time (h) and bile salt concentration \%

Figure 1. Sensitivity of Lactobacillus plantarum ZDY 2013 to acid and bile salts. Growth curve (A; optical density at $600 \mathrm{~nm})$ and pH value of fermentation culture (C) of ZDY 2013 in de Man, Rogosa, Sharpe medium with different initial pH $6.5(\mathbf{\bullet}), 5.0(\mathbf{\square}), 4.0(\boldsymbol{\Delta}), 3.5(\boldsymbol{\nabla})$, and $3.0(\bullet)$; (B) viable counts of ZDY 2013 at extreme $\mathrm{pH} 2.0(\triangle)$ and $3.0(\bigcirc)$; and $(\mathrm{D})$ bile salt resistance of ZDY 2013 in different sample points. Compared with initial value, ${ }^{*} P<0.05 ; * *<0.01$. 


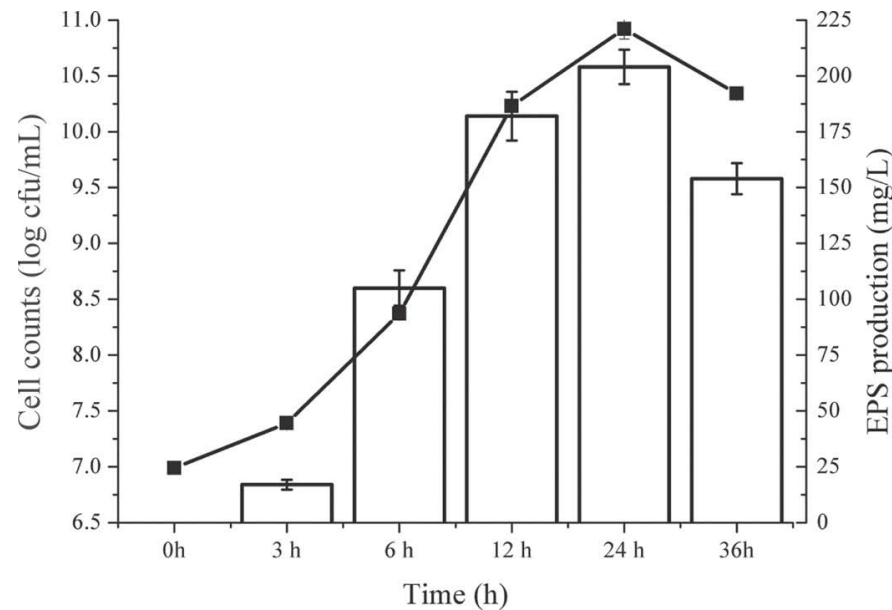

Figure 2. Bacteria cultivation and exopolysaccharide (EPS) production: Strain Lactobacillus plantarum ZDY 2013 was grown in de Man, Rogosa, Sharpe medium, and the cell number (line) and EPS production (column) were tested for a period of $36 \mathrm{~h}$. Error bars represent SD.

\section{Detection of the Major Species in the Intestine or Feces of Mice by $q P C R$}

Principally, L. plantarum ZDY 2013 was a biota of the host. To investigate the exact effect of this organism on the major species of the intestinal- or fecal biota of the mice, we used qPCR for semiquantitative detection of intestinal biotal constituents. After 2 wk of administration of L. plantarum ZDY 2013, the activity, behavior of mice, and the serum biochemical indexes as well as organ indices were normal (data not shown). As shown in Table 3, probiotics (i.e., Lactobacillus and Bifidobacterium) increased by at least 1.43 fold in the colon and cecum of mice; however, the former increased in feces by 1.91 fold and Bifidobacterium decreased by 0.9 fold. The potential enteropathogenic organisms (e.g., Fusobacterium and Bacteroidetes) decreased significantly in

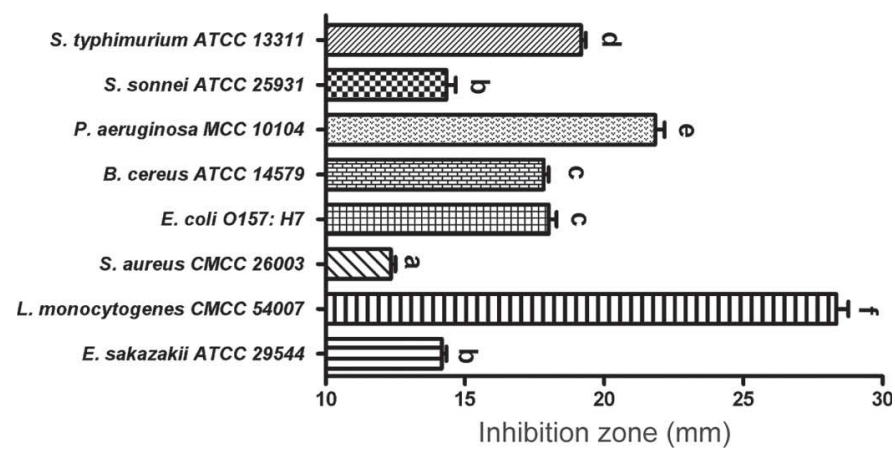

Figure 3. Antibacterial activities of the cultured supertanant of Lactobacillus plantarum ZDY 2013 against 8 kinds of food-borne pathogenic bacteria. Symbols with different letter (a-f) are significantly different at $P<0.05$. Error bars represent SD.
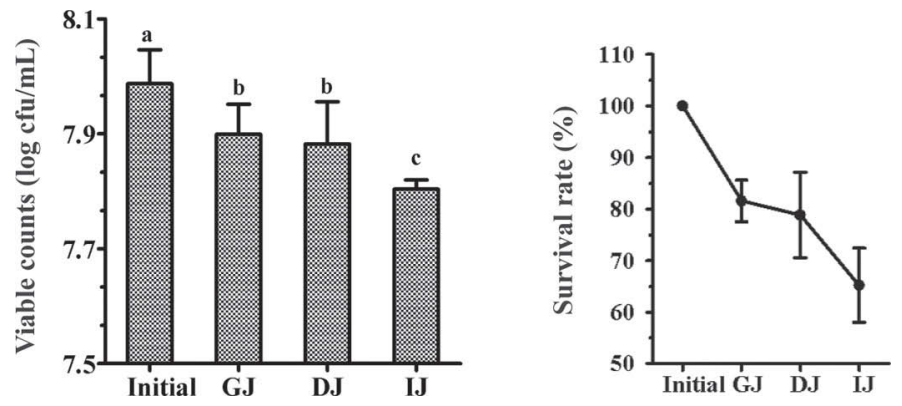

Figure 4. Survival of cell counts and relative survival rate of Lactobacillus plantarum ZDY 2013 in transit under the simulated gastrointestinal tract. Viable counts $(\log \mathrm{cfu} / \mathrm{mL})$ and the percentage of survival rate were obtained at each sampling point. GJ = gastric juice; DJ = duodenal juice; and IJ = intestinal juice. Symbols with different letters $(\mathrm{a}-\mathrm{c})$ are significantly different at $P<0.05$. Error bars represent SD.

the colon; Enterococcus reduced at least by 3.35 fold both in intestinal tract and the feces, whereas both Enterobacterium and Clostridium perfringens decreased in the colon and cecum but increased in the feces.

\section{General Health Status and Hepatic Indices of Mice}

General health status of mice after oral administration with L. plantarum ZDY 2013 is shown in Supplementary Table S1 (http://dx.doi.org/10.3168/jds.20149153) and serum biochemical indices are shown in Supplementary Table S2 (http://dx.doi.org/10.3168/ jds.2014-9153). The BW and organ indices were very similar (Supplementary Table S1). As shown in Supplementary Table S2, the 10 important hepatic indices (alanine aminotransferase, aspartate aminotransferase, total bilirubin, direct bilirubin, total protein, albumin, globulin, ratio of albumin to globulin, gamma glutamyl transaminase, and alkaline phosphatase) were not significantly altered and were within a reasonable range independent of the oral administration of L. plantarum ZDY 2013 in comparison with the control. The indices of the kidney function monitored were creatinine, BUN, and urea, which exhibited similar levels as compared with the control group. A standard range of serum biochemical markers is reported in our previous study (Yang et al., 2015). Therefore, no hepatic and renal abnormity was observed in the animals after $L$. plantarum ZDY 2013 treatment, which suggested that mice were in a good health status with administration of probiotic, L. plantarum ZDY2013.

\section{DGGE Analysis}

To have a detailed overview of the individual biota in mice influenced by oral administration of $L$. plantarum 
Table 3. Fold change of internal microbiota population in colon, cecum, and feces as determined by quantitative $\mathrm{PCR}^{1}$

\begin{tabular}{lrrr}
\hline Sample & \multicolumn{1}{c}{$\Delta \mathrm{Ct}$} & \multicolumn{1}{c}{$\Delta \Delta \mathrm{Ct}$} & $2^{-\Delta \Delta \mathrm{Ct}}$ \\
\hline Colon & & & \\
Enterococcus & $15.36 \pm 1.94$ & $3.88 \pm 1.94$ & $0.068 \pm 0.14$ \\
Lactobacillus & $4.35 \pm 0.59$ & $-0.63 \pm 0.59$ & $1.55 \pm 0.75$ \\
Fusobacterium & $8.29 \pm 0.53$ & $0.56 \pm 0.53$ & $0.68 \pm 0.24$ \\
Enterobacterium & $13.38 \pm 0.57$ & $0.98 \pm 0.58$ & $0.51 \pm 0.24$ \\
Bacteroidetes & $2.64 \pm 0.36$ & $0.56 \pm 0.36$ & $0.68 \pm 0.18$ \\
Bifidobacterium & $21.93 \pm 0.38$ & $-0.88 \pm 0.37$ & $1.84 \pm 0.48$ \\
Clostridium perfringens & $19.81 \pm 0.39$ & $0.99 \pm 0.39$ & $0.51 \pm 0.12$ \\
Cecum & & & \\
Enterococcus & $15.42 \pm 0.41$ & $3.77 \pm 0.41$ & $0.08 \pm 0.02$ \\
Lactobacillus & $4.71 \pm 0.76$ & $-0.65 \pm 0.55$ & $1.73 \pm 0.82$ \\
Fusobacterium & $7.53 \pm 0.44$ & $-0.67 \pm 0.44$ & $1.65 \pm 0.50$ \\
Enterobacterium & $10.40 \pm 0.16$ & $2.03 \pm 0.16$ & $0.25 \pm 0.03$ \\
Bacteroidetes & $2.54 \pm 0.06$ & $0.23 \pm 0.06$ & $1.17 \pm 0.05$ \\
Bifidobacterium & $18.26 \pm 0.48$ & $-0.45 \pm 0.48$ & $1.43 \pm 0.46$ \\
Clostridium perfringens & $17.16 \pm 0.56$ & $0.49 \pm 0.56$ & $0.75 \pm 0.28$ \\
Feces & & & \\
Enterococcus & $13.27 \pm 1.08$ & $2.11 \pm 1.08$ & $0.23 \pm 0.21$ \\
Lactobacillus & $5.40 \pm 0.71$ & $-0.93 \pm 0.71$ & $1.91 \pm 0.19$ \\
Fusobacterium & $8.96 \pm 0.15$ & $-0.74 \pm 0.15$ & $1.67 \pm 0.18$ \\
Enterobacterium & $9.81 \pm 0.18$ & $-0.89 \pm 0.18$ & $1.85 \pm 0.23$ \\
Bacteroidetes & $2.72 \pm 0.24$ & $-1.52 \pm 0.24$ & $2.86 \pm 0.48$ \\
Bifidobacterium & $21.04 \pm 0.43$ & $0.99 \pm 0.43$ & $0.50 \pm 0.15$ \\
Clostridium perfringens & $19.48 \pm 0.30$ & $-1.61 \pm 0.30$ & $3.05 \pm 0.60$ \\
\hline Sampleswer & & & \\
\hline
\end{tabular}

${ }^{1}$ Samples were run in triplicate and represented by mean \pm SD. $\Delta \mathrm{Ct}$ represents the difference between the cycle threshold $(\mathrm{Ct})$ value with the primers to each bacterium and the $\mathrm{Ct}$ value with the primers to total bacteria. $\Delta \Delta \mathrm{Ct}$ represents the difference between the $\Delta \mathrm{Ct}$ value of Lactobacillus plantarum ZDY 2013 group and the $\Delta \mathrm{Ct}$ value of the control. $2^{-\Delta \Delta \mathrm{Ct}}$ are fold changes to represent relative abundance (reference samples of control group $=1.0)$ of the population.

ZDY 2013, 6 samples were collected randomly from colon, cecum, and feces of control and ZDY 2013-challenged groups and examined by PCR-DGGE followed by PCA. The bacterial profiles, PCA plots (Figure 5-A, $\mathrm{B}, \mathrm{C}$ ), and the richness (Figure 5-D) of different parts in mice intestine of the control and ZDY 2013-challenged mice are shown in Figure 5. All the PCA dots in the control group gather together, except for the separation of the number 6 mice in colon and cecum, which illustrates the stability of gut microbiota in mice. However, the PCA plots exhibited the tendency of separation in the profiles after the interference of ZDY 2013 for 14 $\mathrm{d}$, especially in cecum and fecal parts in mice intestine. In addition, the richness which represented the band numbers reduced significantly in the cecal $(P<0.05)$ DGGE profiles, whereas they increased in fecal $(P<$ $0.05)$ but not in colon profiles.

\section{DISCUSSION}

The beneficial effects of probiotics, including modulation of biota balance in GIT, and enhancement of the host immunity have attracted attention of many researchers for novel and potential commercial strains of lactic acid bacteria and bifidobacteria from traditional fermented food. We isolated a novel L. plantarum
ZDY 2013 from Chinese traditional acid beans and systematically evaluated its potential as a probiotic by testing its resistance in extreme acid condition, bile salt concentration, antibiotic sensitivity, and its effects on intestinal biota constituents.

Lactobacillus plantarum, already used in starter culture of yogurt and other products, has been known for resistance to extreme acid and bile salt and could be a potential candidate for use as a probiotic. Lee et al. (2011) reported that several isolates, identified as $L$. plantarum from Korean kimchi, could survive at $\mathrm{pH}$ 2.5 for $2 \mathrm{~h}$. Some L. plantarum maintained $1.0 \times 10^{5}$ $\mathrm{cfu} / \mathrm{mL}$ after a treatment at $\mathrm{pH} 1.5$ for $4 \mathrm{~h}$ (Chen et al., 2014b). Generally, the reduction of cell counts of L. plantarum in various studies was at least one order of magnitude (Anderson et al., 2010; Zago et al., 2011; Guidone et al., 2014). However, we found that L. plantarum ZDY 2013 can survive for $6 \mathrm{~h}$ at $\mathrm{pH} 2.0$ with loss of less than one order of magnitude. Therefore, L. plantarum ZDY 2013 demonstrated better survival in extreme acid environment as compared with other reported strains.

Transit ability during passage through the GIT was the main focus of evaluation of L. plantarum ZDY 2013 for its potential use as a probiotic. In addition to facing challenges of extreme acidity and high concentrations 

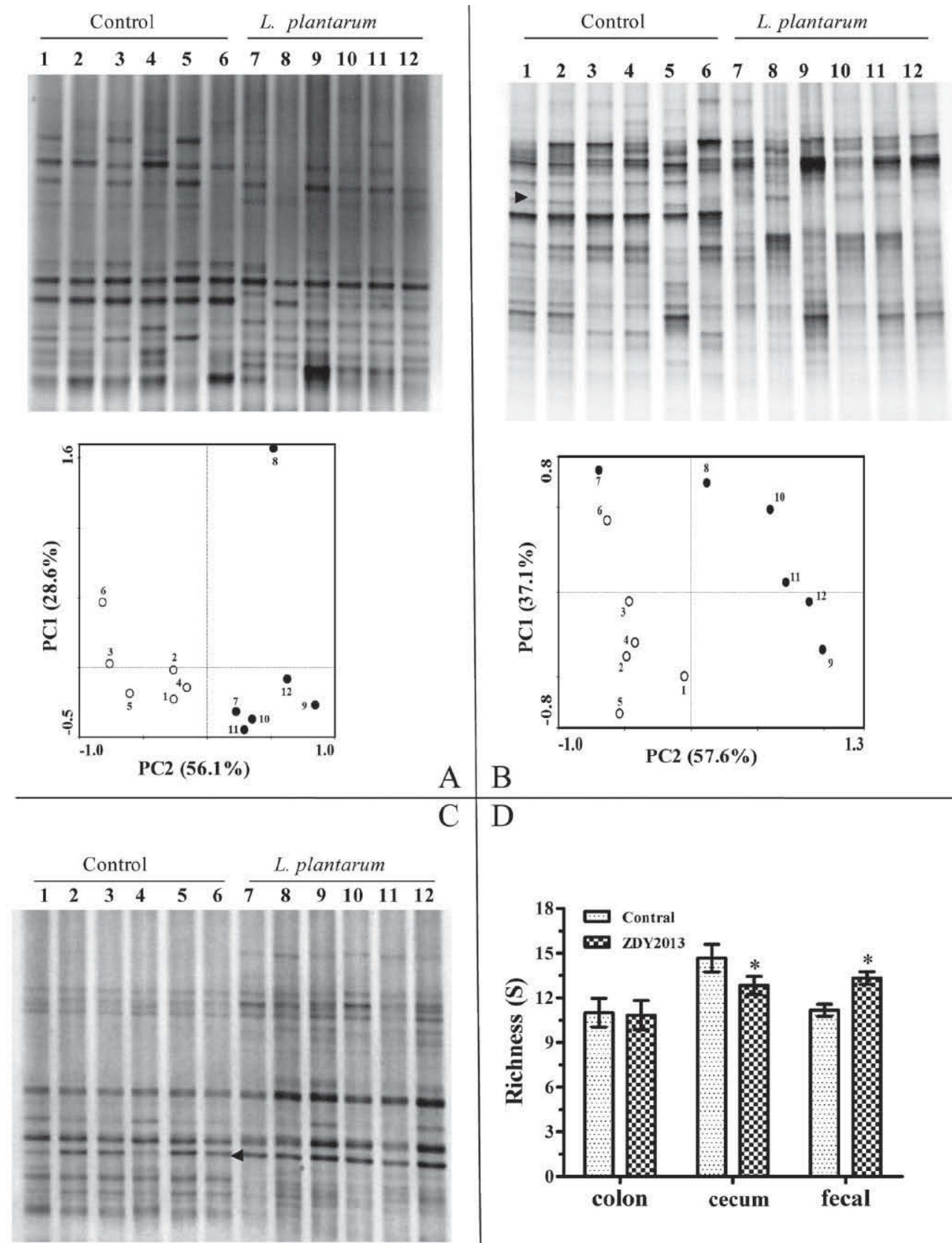

C

\section{B}

$\mathrm{D}$
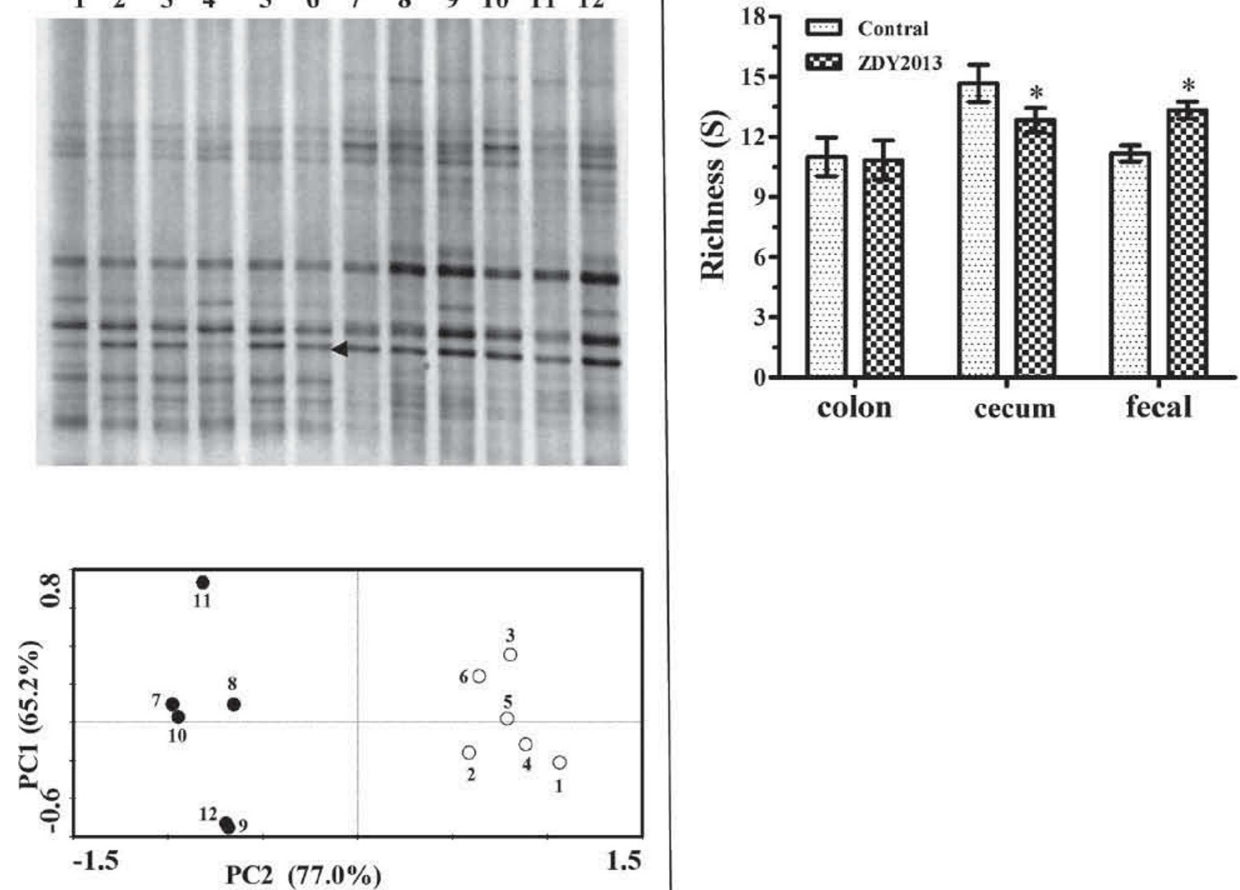

Figure 5. The DGGE fingerprint and principal component analysis of the colon contents (A), cecum contents (B), and feces (C) for the microbial community of mice. 1-6=6 mice for control group; 7-12=6 mice for experimental group of Lactobacillus plantarum ZDY 2013. (D) Richness of intestinal tract and feces microbiota. Compared with control group, ${ }^{*} P<0.05$. Error bars represent SD. 
of bile salt, the survival of probiotics is also affected by the digestive enzymes (pepsin, pancreatin). As demonstrated in our study, as high as $65.84 \%$ of the L. plantarum ZDY 2013 cells could pass through the subsequent challenge of simulated GJ, DJ, and IJ. In contrast, Zago et al. (2011) reported that the reduction in the 9 isolates of L. plantarum from an initial $10^{9} \mathrm{cfu} /$ $\mathrm{mL}$ to a final $10^{6} \mathrm{cfu} / \mathrm{mL}$ in GIT transit. A high level of survival indicated that L. plantarum ZDY 2013 can potentially be orally administrated as a probiotic.

Potential of ZDY 2013 as a probiotic was based not only on survival ability during passage through the GIT, but also by the antagonistic effect against pathogens and in vivo microbial interference. Our data showed that ZDY 2013 inhibited 8 common food-borne pathogens in this order: L. monocytogenes, P. aeruginosa, S. typhimurium, E. coli, B. cereus, S. sonnei, E. sakazakii, and $S$. aureus. In a similar study, Kaushik et al. (2009) reported that the inhibition order was $B$. cereus, L. monocytogenes, and E. coli. Among pathogens, L. monocytogenes was the most sensitive and was inhibited by $L$. plantarum either in diffusion test or coincubation test, as we achieved a halo as large as 28 $\mathrm{mm}$ in diameter against L. monocytogenes. Antagonistic bacterial substances from $L$. plantarum may include bacteriocin (e.g., plantaricin Y) and organic acid (e.g., lactic acid and acetic acid), or even EPS (Crowley et al., 2013; Anas et al., 2014; Chen et al., 2014c; Li et al., 2014b). In our study, we found that the acidification with ZDY 2013 took place even at $\mathrm{pH}$ 3.5; with the growth of cells and accumulation of EPS, those organic acids contributed to the antagonistic effect, which was also documented by Wilson et al. (2005). In our previous study with $L$. plantarum, we reported that L. monocytogenes, S. aureus, and B. cereus were significantly inhibited by EPS produced by L. plantarum R315 (Li et al., 2014b).

High transit efficiency of ZDY 2013 provided a precondition for colonization of intestinal tract, and thereafter resulted in specific host-microbial interactions. In our study, qPCR in vivo analysis of mice showed that the administration of ZDY 2013 had successfully enhanced the native bacterial population (e.g., Bifidobacterium and Lactobacillus groups in colon and cecum) and reduced the potential enteropathogenic bacteria (e.g., Enterococcus, Clostridium perfringens). Its antagonistic effect might affect the binding of pathogenic organisms to the GIT lining and thus help in expediting their clearance from the host. Some studies have also demonstrated that feeding rats with probiotics, prebiotics, or synbiotics promoted increases in Bifidobacterium and Lactobacillus population at different locations in the rat GIT (Tuohy et al., 2003; Quigley, 2010). The DGGE profile demonstrated that the probiotic properties of $L$. plantarum ZDY 2013 may partially depend on its effect on the microbial diversities at various intestinal loci.

Moreover, for therapeutic or prophylactic applications of ZDY 2013, the antibiotic sensitivity of this organism was also investigated and demonstrated that ZDY 2013 was sensitive to amoxicillin, tetracycline, and rifampicin and resistant to bacitracin, ciprofloxacin, and cefoxitin. Whereas L. plantarum ZDY 2013 showed high resistance to bacitracin and intermediate resistance to penicillin, Charteris et al. (1998) reported that 46 Lactobacillus strains from human and dairy sources were assayed for susceptibility to 44 antibiotics; however, only 4 human strains and 1 dairy strain exhibited resistance to bacitracin. Temmerman et al. (2003) reported that antibiotic resistance among 187 isolates was detected, but only $23 \%$ of strains were resistant to penicillin. Lactobacillus plantarum ZDY 2013 originated from natural fermented product and presented specific antibiotic resistance to bacitracin and penicillin, which might be an advantage considering a joint use for probiotic therapy in the future. The information in our study provides a reasonable basis for the application of this probiotic along with certain antibiotics for improving the balance of the host GIT biota.

\section{CONCLUSIONS}

Lactobacillus plantarum ZDY 2013 was systematically evaluated for its survival ability under an extreme acid environment, a high concentration of bile salt, and a simulated GIT in vitro. The interference of ZDY 2013 to the diversity and constituents of biota in mice was monitored by qPCR and PCR-DGGE. The antagonistic effect on pathogens and antibiotic sensitivity as well as EPS yields were also tested in this study. Our results showed that ZDY 2013 was a potential probiotic candidate for its outstanding stress-resistant ability (e.g., survival at $\mathrm{pH} 2.0$ for $6 \mathrm{~h}$ and at $0.45 \%$ bile salt concentration for $3 \mathrm{~h}$, maintaining $65.84 \%$ survival rate after GIT transit, and producing as high as $204 \mathrm{mg} / \mathrm{L}$ of EPS). Lactobacillus plantarum ZDY 2013 showed high resistance to bacitracin and intermediate resistance to penicillin, providing a possibility for using this organism along with certain antibiotics in a reasonable dosage for treating GIT disorders, but this claim needs further study. The results also showed an antagonistic effect on 8 common food-borne pathogens; the effect on the microbial diversities at various intestinal loci was shown in the results of PCR-DGGE and the reduction of potential enteropathogenic bacteria while enhancing Bifidobacterium and Lactobacillus group was confirmed by qPCR. The results suggested that ZDY 2013 could be a probiotic candidate, which might have potential applications in nutraceutical and dairy products. 


\section{ACKNOWLEDGMENTS}

This project was sponsored by the National Natural Science Foundation of China (NSF31170091, 31360377, 31260363), the Ganpo Talent Engineering 555 Project, the Academic and Technical Leaders Training Program for Major Subjects of Jiangxi Province (2009, P.R. China).

\section{REFERENCES}

Anas, M., B. A. Zinedine, H. A. Rizk, H. J. Eddine, and K. Mebrouk. 2014. Screening of autochthonous Lactobacillus species from Algerian raw goats' milk for the production of bacteriocin like compounds against Staphylococcus aureus. Afr. J. Biotechnol. 11:4595-4607.

Anderson, R. C., A. L. Cookson, W. C. McNabb, W. J. Kelly, and N. C. Roy. 2010. Lactobacillus plantarum DSM 2648 is a potential probiotic that enhances intestinal barrier function. FEMS Microbiol. Lett. 309:184-192.

Bartosch, S., A. Fite, G. T. Macfarlane, and M. E. McMurdo. 2004 Characterization of bacterial communities in feces from healthy elderly volunteers and hospitalized elderly patients by using realtime PCR and effects of antibiotic treatment on the fecal microbiota. Appl. Environ. Microbiol. 70:3575-3581.

ben Omar, N., and F. Ampe. 2000. Microbial community dynamics during production of the Mexican fermented maize dough pozol. Appl. Environ. Microbiol. 66:3664-3673.

Borriello, S. P., W. Hammes, W. Holzapfel, P. Marteau, J. Schrezenmeir, M. Vaara, and V. Valtonen. 2003. Safety of probiotics that contain lactobacilli or bifidobacteria. Clin. Infect. Dis. 36:775-780.

Charteris, W. P., P. M. Kelly, L. Morelli, and J. K. Collins. 1998. Antibiotic susceptibility of potentially probiotic Lactobacillus species. J. Food Prot. 61:1636-1643.

Chen, T., S. Jiang, S. Xiong, M. Wang, D. Zhu, and H. Wei. 2012. Application of denaturing gradient gel electrophoresis to microbial diversity analysis in Chinese Douchi. J. Sci. Food Agric. 92:21712176

Chen, T., S. Li, and H. Wei. 2014a. Antibiotic resistance capability of cultured human colonic microbiota growing in a chemostat model. Appl. Biochem. Biotechnol. 173:765-774.

Chen, T., M. Wang, S. Jiang, S. Xiong, and H. Wei. 2013. The application of polymerase chain reaction-denaturing gradient gel electrophoresis (PCR-DGGE) method in microbial screening. Afr. J. Biotechnol. 10:9387-9395.

Chen, T., M. Wang, S. Jiang, S. Xiong, D. Zhu, and H. Wei. 2011. Investigation of the microbial changes during koji-making process of Douchi by culture-dependent techniques and PCR-DGGE. Int. J. Food Sci. Technol. 46:1878-1883.

Chen, T., Q. Wu, S. Li, S. Xiong, S. Jiang, Q. Tan, Z. Zhang, D. Zhu, and H. Wei. 2014b. Microbiological quality and characteristics of probiotic products in China. J. Sci. Food Agric. 94:131-138.

Chen, Y.-S., Y.-C. Wang, Y.-S. Chow, F. Yanagida, C.-C. Liao, and C.-M. Chiu. 2014c. Purification and characterization of plantaricin Y, a novel bacteriocin produced by Lactobacillus plantarum 510 . Arch. Microbiol. 196:193-199.

Cheng, L., J. Luo, P. Li, H. Yu, J. Huang, and L. Luo. 2014. Microbial diversity and flavor formation in onion fermentation. Food Funct. 5:2338-2347

Crowley, S., J. Mahony, and D. van Sinderen. 2013. Broad-spectrum antifungal-producing lactic acid bacteria and their application in fruit models. Folia Microbiol. (Praha) 58:291-299.

Feng, Y., J. Gong, H. Yu, Y. Jin, J. Zhu, and Y. Han. 2010. Identification of changes in the composition of ileal bacterial microbiota of broiler chickens infected with Clostridium perfringens. Vet. Microbiol. 140:116-121.

Fooks, L. J., and G. R. Gibson. 2002. Probiotics as modulators of the gut biota. Br. J. Nutr. 88(Suppl. 1):S39-49
García-Ródenas, C. L., G. E. Bergonzelli, S. Nutten, A. Schumann, C. Cherbut, M. Turini, K. Ornstein, F. Rochat, and I. CorthésyTheulaz. 2006. Nutritional approach to restore impaired intestinal barrier function and growth after neonatal stress in rats. J. Pediatr. Gastroenterol. Nutr. 43:16-24.

Gill, H. S. 2004. Probiotics and human health: a clinical perspective. Postgrad. Med. J. 80:516-526.

Guidone, A., T. Zotta, R. P. Ross, C. Stanton, M. C. Rea, E. Parente, and A. Ricciardi. 2014. Functional properties of Lactobacillus plantarum strains: A multivariate screening study. LWT Food Sci. Technol. 56:69-76.

Jankovic, I., W. Sybesma, P. Phothirath, E. Ananta, and A. Mercenier. 2010. Application of probiotics in food products - Challenges and new approaches. Curr. Opin. Biotechnol. 21:175-181.

Kaushik, J. K., A. Kumar, R. K. Duary, A. K. Mohanty, S. Grover, and V. K. Batish. 2009. Functional and probiotic attributes of an indigenous isolate of Lactobacillus plantarum. PLoS ONE 4:e8099.

Kleerebezem, M., J. Boekhorst, R. van Kranenburg, D. Molenaar, O. P. Kuipers, R. Leer, R. Tarchini, S. A. Peters, H. M. Sandbrink, and M. W. Fiers. 2003. Complete genome sequence of Lactobacillus plantarum WCFS1. Proc. Natl. Acad. Sci. USA 100:1990-1995. http://dx.doi.org/10.1073/pnas.0337704100.

Lee, J., H. S. Yun, K. W. Cho, S. Oh, S. H. Kim, T. Chun, B. Kim, and K. Y. Whang. 2011. Evaluation of probiotic characteristics of newly isolated Lactobacillus spp.: Immune modulation and longevity. Int. J. Food Microbiol. 148:80-86.

Li, S., T. Chen, F. Xu, S. Dong, H. Xu, Y. Xiong, and H. Wei. 2014a. The beneficial effect of exopolysaccharides from Bifidobacterium bifidum WBIN03 on microbial diversity in mouse intestine. J. Sci. Food Agric. 94:256-264.

Li, S., R. Huang, N. P. Shah, X. Tao, Y. Xiong, and H. Wei. 2014b. Antioxidant and antibacterial activities of exopolysaccharides from Bifidobacterium bifidum WBIN03 and Lactobacillus plantarum R315. J. Dairy Sci. 97:7334-7343.

Liong, M. T., and N. P. Shah. 2005. Acid and bile tolerance and cholesterol removal ability of lactobacilli strains. J. Dairy Sci 88:55-66.

Luo, H., H. Qi, and H. Zhang. 2004. Assessment of the bacterial diversity in fenvalerate-treated soil. World J. Microbiol. Biotechnol. 20:509-515.

Naidu, A. S., W. Bidlack, and R. Clemens. 1999. Probiotic spectra of lactic acid bacteria (LAB). Crit. Rev. Food Sci. Nutr. 39:13-126.

Parracho, H., A. L. McCartney, and G. R. Gibson. 2007. Probiotics and prebiotics in infant nutrition. Proc. Nutr. Soc. 66:405-411.

Parvez, S., K. A. Malik, S. Ah Kang, and H. Y. Kim. 2006. Probiotics and their fermented food products are beneficial for health. J. Appl. Microbiol. 100:1171-1185.

Quigley, E. M. 2010. Prebiotics and probiotics; modifying and mining the microbiota. Pharmacological research . Off. J. Ital. Pharm. Soc. $61: 213-218$

Rinttilä, T., A. Kassinen, E. Malinen, L. Krogius, and A. Palva. 2004 Development of an extensive set of $16 \mathrm{~S}$ rDNA-targeted primers for quantification of pathogenic and indigenous bacteria in faecal samples by real-time PCR. J. Appl. Microbiol. 97:1166-1177.

Salazar, N., A. Binetti, M. Gueimonde, A. Alonso, P. Garrido, C. Gonzalez del Rey, C. Gonzalez, P. Ruas-Madiedo, and C. G. de los Reyes-Gavilan. 2011. Safety and intestinal microbiota modulation by the exopolysaccharide-producing strains Bifidobacterium animalis IPLA R1 and Bifidobacterium longum IPLA E44 orally administered to Wistar rats. Int. J. Food Microbiol. 144:342-351.

Salminen, S., A. von Wright, and L. Morelli. 1998. Demonstration of safety of probiotics - A review. Int. J. Food Microbiol. 44:93-106.

Tamime, A. Y. 2002. Fermented milks: A historical food with modern applications. Eur. J. Clin. Nutr. 56(Suppl 4):S2-S15.

Tan, Q., H. Xu, Z. P. Aguilar, S. Peng, S. Dong, B. Wang, P. Li, T. Chen, F. Xu, and H. Wei. 2013. Safety assessment and probiotic evaluation of Enterococcus faecium YF5 isolated from sourdough. J. Food Sci. 78:M587-M593.

Temmerman, R., B. Pot, G. Huys, and J. Swings. 2003. Identification and antibiotic susceptibility of bacterial isolates from probiotic products. Int. J. Food Microbiol. 81:1-10. 
Tuohy, K. M., H. M. Probert, C. W. Smejkal, and G. R. Gibson. 2003. Using probiotics and prebiotics to improve gut health. Drug Discov. Today 8:692-700.

Wang, L., P. Li, Z. Zhang, Q. Chen, Z. P. Aguilar, H. Xu, L. Yang, F. Xu, W. Lai, Y. Xiong, and H. Wei. 2014. Rapid and accurate detection of viable Escherichia coli $\mathrm{O} 157: \mathrm{H} 7$ in milk using a combined IMS, sodium deoxycholate, PMA and real-time quantitative PCR process. Food Contr. 36:119-125.

Wei, H., G. Wolf, and W. P. Hammes. 2006. Indigenous microorganism from iceberg lettuce with adherence and antagonist potential for use protective culture. Innov. Food Sci. Emerg. Technol. $7: 294-301$.

Wilson, A. R., D. Sigee, and H. Epton. 2005. Anti-bacterial activity of Lactobacillus plantarum strain SK1 against Listeria monocytogenes is due to lactic acid production. J. Appl. Microbiol. 99:1516-1522.
Wise, M. G., and G. R. Siragusa. 2005. Quantitative detection of Clostridium perfringens in the broiler fowl gastrointestinal tract by real-time PCR. Appl. Environ. Microbiol. 71:3911-3916.

Wu, R., L. Wang, J. Wang, H. Li, B. Menghe, J. Wu, M. Guo, and H. Zhang. 2009. Isolation and preliminary probiotic selection of lactobacilli from koumiss in Inner Mongolia. J. Basic Microbiol. 49:318-326.

Yang, L., H. Kuang, W. Zhang, Z. P. Aguilar, Y. Xiong, W. Lai, H. $\mathrm{Xu}$, and H. Wei. 2015. Size dependent biodistribution and toxicokinetics of iron oxide magnetic nanoparticles in mice . Nanoscale 7:625-636.

Zago, M., M. E. Fornasari, D. Carminati, P. Burns, V. Suàrez, G. Vinderola, J. Reinheimer, and G. Giraffa. 2011. Characterization and probiotic potential of Lactobacillus plantarum strains isolated from cheeses. Food Microbiol. 28:1033-1040. 